apresentação de caso

\author{
Fredevico F.R. Maia \\ Levimar R. Araújo
}

Clínica de Endocrinologia e

Metabologia, Hospital

Universitário São José - Faculdade de

Ciências Médicas de Minas Gerais, Belo

Horizonte, $M G$.

\title{
Sindrome de Mauriac: Forma Rara do Diabetes Mellitus Tipo 1
}

RESUMO

O diabetes mellitus tipo 1 (DM1) é uma das doenças endócrinometabólicas mais importantes na faixa etária pediátrica, podendo se manifestar com alterações sistêmicas decorrentes do controle glicêmico inadequado, como hepatomegalia e déficit de crescimento. Apresentamos o caso de uma criança com DM1 descompensado, evoluindo com sinais clínicos e laboratoriais sugestivos de síndrome de Mauriac. Alertamos para a importância do conhecimento dessa forma rara de DM1, buscando orientar e esclarecer dúvidas dos profissionais a respeito das condutas propedêuticas e terapêuticas na síndrome de Mauriac. (Arq Bras Endocrinol Metab 2002;46/3:310-315)

Descritores: Diabetes mellitus tipo 1; Glicogenose diabética; Hepatomegalia; Síndrome de Mauriac

\section{ABSTRACT}

Mauriac's Syndrome: Uncommon Form of Type 1 Diabetes Mellitus.

Type 1 diabetes mellitus (DM1) is one of the most important pediatric endocrinology diseases. It has different kinds of manifestation, such as systemic disturbances caused by poor glycemic control, like hepatomegaly and dwarfism. The outcome of 8 years of poor glycemic control with clinical and laboratory signs suggesting Mauriac's syndrome is presented. This is an uncommon form of DM1. The aim of this study is to update, guide and answer some doubts of professionals with regard to diagnosis and treatment of cases of Mauriac's syndrome. (Arq Bras Endocrinol Metab 2002;46/3:310-315)

Keywords: Diabetes mellitus type 1; Diabetic glucogenosis; Hepatomegaly; Mauriac's syndrome

$\mathbf{A}^{\mathrm{s}}$ SíndRome De MaURiac (SM) é uma forma rara de diabetes mellitus tipo $1(\mathrm{DMl})$, caracterizada pela sintomalogia tríplice de hepatomegalia, retardo do crescimento e diabetes mal controlado de longa evolução. É um estado pouco comum que acomete principalmente crianças e adolescentes portadores da doença, com difícil controle glicêmico (1).

Estima-se que $1 \mathrm{em} 2500$ crianças com idade inferior a 5 anos e $1 \mathrm{em}$ 300 pessoas abaixo de 18 anos são portadoras dessa doença (2). Cerca de $60 \%$ dos DMl com controle glicêmico inadequado evoluem com hepatomegalia associada (1). A real incidência da SM ainda não está bem definida, em função do reduzido número de casos descritos na literatura mundial.

O quadro clínico, em geral, cursa com déficit pôndero-estatural, obesidade, dor em flanco direito associada a náuseas e vômitos secundários à hepatomegalia (3). O crescimento do fígado é variável de acordo com a debilidade do controle glicêmico do paciente. Ainda não se pode quantificar o grau de deficiência do controle glicêmico em crianças com DMl que determina o aparecimento desse quadro (4). As repercussões sobre o
Recebido em 19/11/01

Revisado em 04/02/02

Aceito em 08/03/02 
desenvolvimento do indivíduo e o mecanismo exato dessa síndrome ainda não estão bem estabelecidos.

Apresentamos um caso de uma criança diabética insulino-dependente evoluindo com hepatomegalia grave e atraso do desenvolvimento pôndero-estatural. Objetiva-se alertar a classe médica da importância do diagnóstico precoce dessa síndrome, discutindo-se as condutas propedêuticas e terapêuticas na SM.

\section{RELATO DO CASO}

LPL, 13 anos, masculino, leucoderma, natural e procedente de Itabira (MG). Aos 5 anos de idade apresentou quadro agudo de polidipsia, poliúria e perda de peso seguido de internação hospitalar em quadro compatível com cetoacidose diabética. Após a confirmação diagnóstica de DMl, foi iniciada insulinoterapia convencional.

Ao longo de oito anos, evoluiu com níveis glicêmicos irregulares e crises constantes de hipoglicemia, encaminhado para avaliação endocrinológica em Junho de 2000. Nessa ocasião, o paciente encontrava-se em uso de insulina NPH em quatro doses diárias, sendo 5UI pela manhã, almoço e jantar e 3UI à noite, associadas à insulina regular em três doses diárias de 5UI pela manhã, almoço e jantar. Ao exame clínico, apresentava atraso de desenvolvimento pôndero-estatural, com altura de $132,5 \mathrm{~cm}$ (percentil $<2,5$ ), peso de $32 \mathrm{~kg}$ (percentil $<2,5$ ) e índice de massa corporal (IMC) de 18,2 $\mathrm{kg} / \mathrm{m}^{2}$, sem demais alterações significativas.

A propedêutica laboratorial evidenciou glicemia de jejum de $127 \mathrm{mg} / \mathrm{dL}$ (VN: 70-110) e glico-hemoglobina (HbAlc) de 8,5\% (VN: <7,9\%). Optou-se pela correção do presente esquema terapêutico de insulina, seguido de acompanhamento por nutricionista e retorno mensal para avaliação do quadro.

$\mathrm{Na}$ história pregressa, a criança apresentava desenvolvimento neuro-psico-motor dentro dos padrões de normalidade. Os pais enfatizaram ausência de déficit intelectual da criança com boa progressão escolar, porém com dificuldade de desenvolvimento físico. Ausência de consagüinidade. Nenhum registro de doença familiar ou supostamente hereditária. $\mathrm{O}$ paciente não fazia uso de nenhuma outra medicação e possuía passado de história vacinal completa.

Nas consultas mensais subseqüentes, o paciente evoluiu com parâmetros clínicos e laboratoriais irregulares, nictúria, difícil controle glicêmico, déficit de crescimento mesmo com o ajuste mensal de dose de insulina e dieta adequada. Após 2 meses, o paciente apresentou HbAlc de $8,0 \%$ e após 4 , os exames de rotina demonstraram glicemia de jejum de $167 \mathrm{mg} / \mathrm{dL}$, HbAlc de 5,8\%, uréia sérica de $29 \mathrm{mmol} / \mathrm{L}$ (VN: 7,0-14,5), creatinina de $0,9 \mathrm{mg} / \mathrm{dL}$ (VN: $0,3-0,7$ ) e proteína urinária de $148 \mathrm{mg} / 24 \mathrm{~h}$ (VN: 28-141). Diante do quadro de microalbuminúria detectada, iniciou-se o tratamento com maleato de enalapril $(5 \mathrm{mg})$, duas vezes ao dia, seguido de novo reajuste de dosagem de insulina. Nessa ocasião, o paciente apresentou pressão arterial sistêmica em níveis fisiológicos (PA: $130 / 85 \mathrm{mmHg}$ ).

Em Março de 2001 o paciente retornou, queixando-se de crescimento progressivo de massa abdominal dolorosa em região de hipocôndrio direito. Foi submetido a novos exames laboratoriais que revelaram função hepática alterada, com elevação dos níveis plasmáticos de TGO: 47U/L (VN: 12-46) e TGP: 74U/L (VN: 10-60), aumento dos níveis de globulinas e gama-GT: 104U/L (VN: 10-50), associados à redução de IGF-l: 36,2ng/ml (VN: 202-957). O laudo ultra-sonográfico de massa abdominal evidenciou "glicogenose diabética". Foi proposto novo esquema insulínico.

Após 2 meses, foi realizada nova avaliação, com dosagem de HbAlc de 10,9 (VN: <7,9\%), comprovando a irregularidade de controle glicêmico do paciente. A radiografia de mãos e punhos revelou idade óssea de 10 anos, confirmando o atraso de desenvolvimento pôndero-estatural do paciente. A fundoscopia demonstrou presença de retinopatia não proliferativa muito leve.

Em agosto de 2001, houve agravamento do quadro clínico com dor intensa em flanco direito, náuseas, vômitos e mal estar geral, associados à dificuldade de controle glicêmico e déficit de crescimento. Ao exame físico, mostrava-se consciente, ativo, hipocorado, anictérico e afebril, medindo $133,5 \mathrm{~cm}$ de altura e $32 \mathrm{~kg}$ de peso, com atraso de desenvolvimento puberal. $\mathrm{O}$ exame do abdome evidenciou abdome tenso, doloroso à palpação profunda e superficial em região de hipocôndrio direito, com fígado palpável a $5 \mathrm{~cm}$ da reborda costal direita, de consistência firme e borda lisa.

Novos exames laboratoriais revelaram: colesterol total de $207 \mathrm{mg} / \mathrm{dL}$ (VN: <170), VLDL: $35 \mathrm{mg} / \mathrm{dL}$ (VN: <30), HDL: 4l $\mathrm{mg} / \mathrm{dL}$ (VN: >35), triglicérides: $175 \mathrm{mg} / \mathrm{dL}$ (VN: <130); glicemia de jejum: $481 \mathrm{mg} / \mathrm{dL}$ e HbAlc: 9,2\%. A análise da função hepática mostrou TGO: 32U/L (VN: 12-46); TGP: 29U/L (VN: 10-60); gama-GT: 40U/L (VN: 10-50), além de redução de IGF-1: 100ng/mL (VN: 202957). Os marcadores de hepatite $B$ foram negativos (HbsAg e anti-Hbe) e as provas de função tireoideana encontravam-se em níveis fisiológicos. O US realizado em setembro levou à conclusão de tratar-se de hepatomegalia secundária à glicogenose (figura 1 ). 


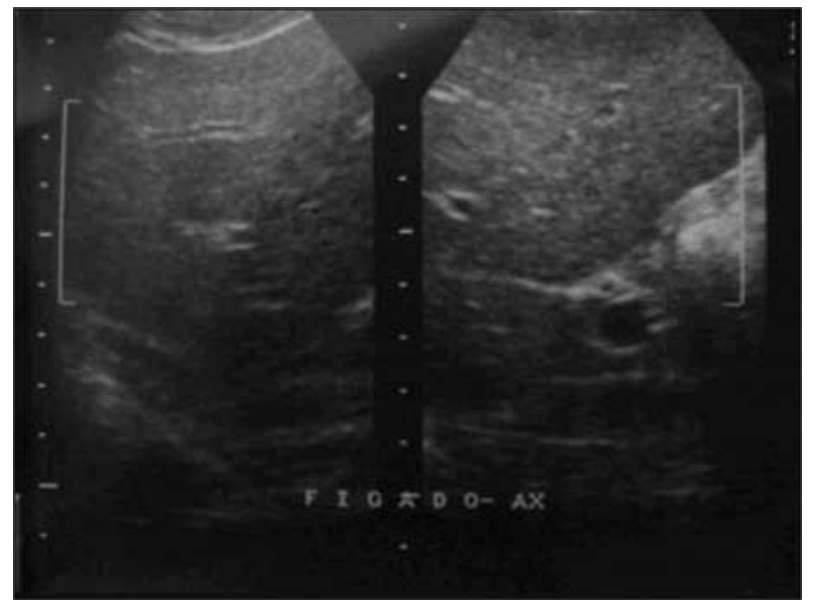

Figura 1. Ultra-sonografia mostrando fígado de volume aumentado, contornos regulares, parênquima homogêneo com aumento difuso de ecogenicidade e da atenuação sônica posterior, sugestivo de glicogenose.

A presença de DMl descompensado de longa evolução e difícil controle, déficit de crescimento, hepatomegalia associados à elevação de transaminases, hipercolesterolemia e redução de IGF-1 indicavam SM.

O tratamento consistiu de terapia insulínica intensiva, atividade física controlada e dieta. Iniciouse esquema terapêutico de multidoses de insulina diariamente, sendo indicado o uso de bomba de infusão de insulina.

Observou-se uma melhora do controle glicêmico, redução das crises hipoglicêmicas, regressão discreta da hepatomegalia, melhora da auto-estima com maior qualidade de vida do paciente. Após três meses de tratamento intensivo, verificou-se uma melhora relativa de desenvolvimento físico, com altura de $134,5 \mathrm{~cm} \mathrm{e}$ $33,5 \mathrm{~kg}$ de peso. Um maior tempo de seguimento permitirá evidenciar melhor tais fatores, sobretudo em relação ao padrão de crescimento do paciente.

\section{DISCUSSÃO}

O referido caso preenche os critérios necessários ao diagnóstico de SM, cursando com retardo de crescimento e hepatomegalia em paciente com DMl descontrolado de longa data, associados às alterações laboratoriais sugestivas desse quadro, como hipercolesterolemia e redução de IGF-1 $(5,6)$.

$\mathrm{O}$ desenvolvimento neurológico e psíquico preservados com déficit de crescimento físico são achados freqüentes nessa síndrome, o que é corroborado pela literatura (5-7). Na SM há acentuada redução da velocidade de crescimento, limitação da mobilidade articular, presença de hepatomegalia, abdome distendido e glo- boso com maior tendência às complicações microangiopáticas como retinopatia e nefropatia. Segundo a literatura, a retinopatia diabética está presente em quase $100 \%$ dos casos quando já há comprometimento renal (8). No caso descrito, o tempo de diabetes, o mau controle glicêmico e a presença de lesão renal foram fatores importantes no surgimento da retinopatia simples, o que é corroborado pela literatura (8).

Um estudo realizado no Brasil acompanhou o crescimento físico de 72 pacientes com DMl com idade média de 21 anos e tempo de diabetes de 11,9 anos. Observaram que a baixa estatura em pacientes com DMl está constantemente associada ao controle glicêmico inadequado (9).

O crescimento hepático é um dos sinais mais importantes nesses casos, sendo de grande relevância clínica. Yokoyama e cols. (10) relataram que a hepatomegalia grave concomitante ao diabetes mal controlado é apanágio de SM. Nessa situação, o fígado pode tornar-se grande, doloroso à palpação, de consistência firme e borda lisa, como visto no presente caso. Um estudo envolvendo pacientes com DMl evidenciou a presença de hepatomegalia em $9 \%$ dos pacientes bem controlados, $60 \%$ dos indivíduos com DM descompensado e $100 \%$ naqueles com cetose (1). Diversos fatores podem ocasionar um aumento progressivo e doloroso do fígado, devendo sempre se excluir causas infecciosas, como hepatites e tuberculose. A história de passado vacinal completo, ausência de quadro clínico sugestivo de processo infeccioso e exames laboratoriais (HbsAg e anti-HBe) negativos foram critérios de exclusão para doença infecciosa nesse caso (11).

Outras situações que freqüentemente cursam com crescimento hepático são os distúrbios metabólicos, doenças hematológicas e do armazenamento. Uma das causas mais comuns de hepatomegalia são as glicogenoses. Na doença de Von Gierke (glicogenose tipo I) e na doença de Cori (glicogenose tipo III) há aumento pronunciado desse órgão. Pode estar presente também em casos de infiltração esteatótica, como no Kwashiorkor e na peliose hepática (12). Polack e cols. (13) descreveram a associação entre desnutrição e SM.

O aumento do fígado no DM se deve ao acúmulo de glicogênio ocasionado pela hiperglicemia secundária ao mau controle glicêmico. A deficiência de insulina com conseqüente elevação da glicose sangüínea impede o feedback necessário para a inibição da gliconeogênese hepática. Com isso, o fígado passaria a produzir uma quantidade ainda maior de glicose, levando a uma enorme disponibilidade dessa substância no sangue. Grande parte dessa glicose será 
convertida em glicogênio e, conseqüentemente, armazenada no fígado (7). Além disso, há um aumento da atividade enzimática da glicose 6 fosfatase no $\mathrm{DMl}$, o que contribui para a maior saída de glicose da célula hepática para o sangue, mesmo nesses estados de hiperglicemia intensa. As enzimas responsáveis pela fixação da glicose nas células, em especial as hexoquinases e as glucoquinases, estão reduzidas, o que acarreta uma maior dificuldade de eliminação da glicose do fígado, culminando com o acúmulo de glicogênio, levando à glicogenose diabética (14).

No diabetes infantil, o aumento do fígado é um sinal de controle glicêmico inadequado e indica infiltração gordurosa e glicogênica, com predomínio dessa sobre aquela (14). A presença de glicogenose hepática e DMl tem sido relacionada com outras entidades clínicas, como a desnutrição por Kwashiorkor em fase de recuperação, tuberculose, septicemia, cirrose, hepatite e doença de Wilson (12).

Nos casos indefinidos clinicamente, a avaliação através de um método de imagem é bastante eficaz. Nesse caso, foi realizada a ultra-sonografia que evidenciou um quadro de glicogenose diabética. Em um caso semelhante, Pashkova e cols. (15) descreveram um achado de hepatomegalia com diagnóstico ultra-sonográfico de glicogenose em paciente de 31 anos de idade com DMl descompensado ao longo de toda vida. Na presença desses sinais, suspeitou-se tratar de SM. A comprovação diagnóstica se deu pela necrópsia. Esse fato nos remete à relevância do diagnóstico de glicogenose ao ultra-som em pacientes com DMl mal controlados, devendo-se aventar a possibilidade dessa síndrome, o que influi diretamente no prognóstico e sobrevida do paciente $(12,15)$.

A biópsia hepática está indicada apenas nos casos mais graves em que o diagnóstico não esteja bem estabelecido. Em 1981, Lorenz e cols. (11) analisaram os resultados de 28 biópsias hepáticas de 19 pacientes com SM. Evidenciaram a presença de glicogenose diabética em todos os casos. Concluíram que a hepatomegalia com glicogenose em achado de biópsia, isoladamente, não é patognomônica da SM. Mas, na presença de DMl descompensado e retardo do crescimento deve ser sempre aventada a possibilidade dessa síndrome.

O diagnóstico de SM é eminentemente clínico, uma vez que não se encontram disponíveis marcadores específicos para essa entidade clínico-patológica. A anamnese e o exame físico apurados são essenciais para a avaliação do paciente, associados aos dados laboratoriais de hipercolesterolemia, provas de função hepática alteradas e redução dos níveis séricos de IGF-1, como detectados no presente relato $(6,9,11)$.
A dosagem de HbAlc é um importante parâmetro de avaliação do paciente com DMl. Segundo o DCCT (Diabetes Control and Complications Trial), o controle glicêmico ideal consiste em níveis de $\mathrm{HbAlc}$ entre 2,8 e 5,2\%. Dosagens de glico-hemoglobina acima de $7,9 \%$ são indicativos de controle metabólico inadequado (16). No referido caso, quatro das cinco medidas de HbAlc realizadas estavam acima de 7,9\%, evidenciando o mau controle glicêmico do paciente.

Mauras e cols. (17) analisaram as variações do IGF-1 em dois pacientes com SM, comparando os resultados com dois outros pacientes com DMl com níveis glicêmicos semelhantes e padrão de crescimento adequado. Foi analisada a atividade pulsátil do $\mathrm{GH}$ durante o período de 24 às 6 horas. Não houve diferença significativa entre os dois grupos. Detectou-se um paciente com $S M$ com resposta deficiente à terapia com GH exógeno. Concluíram que na SM pode haver níveis hormonais normais de GHBP, IGF-1 e IGFBPs e provável resistência aumentada ao receptor de $\mathrm{GH}$ exógeno associada a um defeito na ação de IGF-1 ou resistência a esse receptor.

Outras formas raras de diabetes foram descritas em diversas partes do mundo. Em 1955, Huge-Jones descreveu $13(6 \%)$ casos de DM tipo J, na Jamaica. Nesses casos, o DMl cursa com resistência periférica à insulina aumentada, sendo necessário o uso de doses superiores a $80 \mathrm{U} /$ dia de insulina. Outros casos documentados foram de DMl associado à tireotoxicose, ao feocromocitoma e à síndrome de Cushing (18). Formas incomuns e ainda pouco conhecidas como o diabetes relacionado à mal nutrição, o diabetes pancreático fibrocalculoso e a síndrome da pancreatite tropical juvenil (Indonésia, 1959) também são relatados na literatura $(12,19,20)$.

O prognóstico é reservado e o diagnóstico deve ser o mais precoce possível para que se estabeleça o tratamento intensivo em tempo hábil, evitando as lesões a longo prazo do DM nesse grupo de pacientes (6). Traismam e cols. (21) reportaram o acompanhamento de uma paciente com SM durante 22 anos. Apesar do crescimento deficitário, obtiveram bons resultados quanto ao desenvolvimento psicomotor e sexual, inclusive com duas gestações com sucesso após o controle insulínico e glicêmico adequados. Não foram observadas complicações degenerativas a longo prazo. Tais dados são compatíveis com os resultados do maior estudo epidemiológico envolvendo DMl, o DCCT, em que se verificou que o tratamento insulínico bem realizado acompanhado de dieta e atividade física adequada estão associados a menor risco de complicações crônicas da doença (22). 
O tratamento da SM é o mesmo realizado para as formas tradicionais de $\mathrm{DMl}$, porém deve haver um controle insulínico altamente rígido com monitorização constante dos padrões glicêmicos do indivíduo (23). O início do tratamento deve ser cauteloso, através do ajuste da dosagem de insulina necessária ao longo da terapêutica, principalmente quando já houver retinopatia associada.

Um estudo em 1981 apresentou 4 casos de SM com presença de retinopatia progressiva. $\mathrm{O}$ diagnóstico de SM foi firmado após 7-14 anos de tratamento irregular de DMl. Nessa ocasião, $100 \%$ apresentaram retardo de crescimento, $50 \%$ hepatomegalia e $75 \%$ puberdade tardia. À fundoscopia, detectou-se a presença de microaneurisma em dois casos e hemorragia intrarretiniana em um paciente. Através do controle glicêmico rígido, observaram uma aceleração do padrão de crescimento, regressão da hepatomegalia e desenvolvimento puberal acentuado. Porém, observou-se um agravamento progressivo da retinopatia nos 3 pacientes mais velhos, com aparecimento rápido de microaneurismas, infarto do nervo óptico, hemorragias, exsudatos e edema de mácula. Em um caso, houve hemorragia vítrea com evolução para anopsia esquerda completa. Os autores alertam para a importância da monitorização da função ocular ao iniciar o tratamento insulínico intensivo e correção glicêmica em pacientes com SM (23).

Nos dias de hoje, uma possível solução para o controle glicêmico irregular na SM é o uso da infusão contínua por bomba de insulina. Diversos estudos evidenciaram que esse método, por ser mais fisiológico, vem apresentando melhor controle glicêmico, redução do risco de hipoglicemia, maior aceitação pelo paciente, ocasionando maior satisfação e melhoria da qualidade de vida de pacientes com DMl (24).

Não foi encontrada nenhuma referência na literatura médica sobre o uso da bomba de infusão de insulina em pacientes com SM. No caso descrito, há indicação para o uso da terapia por infusão contínua com bomba de insulina, na busca de um controle metabólico mais rígido e eficaz.

Apesar de rara em nosso meio, consideramos de grande importância que o clínico esteja familiarizado com as alterações hepáticas no DMl. É imperioso que se suspeite de SM em crianças com DMl descompensado, apresentando hepatomegalia grave, retardo do crescimento e achados de hipercolesterolemia com redução de IGF-1. Assim, possibilita-se o diagnóstico precoce dessa síndrome, permitindo-se instituir o tratamento adequado em tempo hábil, reduzindo as complicações agudas e crônicas da doença, com melhora da qualidade de vida do paciente.

\section{REFERÊNCIAS}

1. Sherlock S. O fígado nos distúrbios metabólicos. In: Sherlock S, editor. Doenças do fígado e do sistema biliar. Guanabara-Koogan:Rio de Janeiro, $5^{\mathrm{a}}$ ed., 1978.p.37584.

2. Collet-Solberg PF. Cetoacidose diabética em crianças: Revisão da fisiopatologia e tratamento com o uso do "método de duas soluções salinas". J Ped 2001;77:9-16.

3. Tulzer W, Ploier R. Pathogenic investigations on a case of Mauriac's syndrome. Pediatr Pathol 1976; 1 1:356-63.

4. Lacerda SNL. Doenças metabólicas. In: Dani R, Castro JP, eds. Gastroenterologia clínica. GuanabaraKoogan:Rio de Janeiro, $4^{\mathrm{a}}$ ed, 1981.p.816-7.

5. Silva $A O$. Ultra-som no fígado. In: Silva AO, editor. Hepatologia clínica e cirúrgica. Savier:São Paulo. 1980.p. 113-4.

6. Dorchy $H$, Van VG. Cause of dwarfism in Mauriac's syndrome. J Pediatr 1981;98:857-8.

7. Pupo AA. Diabetes mellito na criança e no adolescente. In: Endocrinologia Pediátrica. São Paulo:Savier. 1989.p.207-8.

8. Aiello LP, Gardner TW, King GL. Diabetic retinopathy. Diabetes Care 1998;21:143-56.

9. Rodrigues TMB, Silva IN. Estatura final de pacientes com diabetes mellitus tipo 1. Arq Bras Endocrinol Metab 2001;45:108-14

10. Yokoyama M, Noto $Y$, Kida H. Hypotermia with acute renal failure in a patient suffering from diabetic nephropathy and malnutrition. Diabetes Metab 2000;26:145-7.

11. Lorenz $G$. Bioptical liver changes in Mauriac's syndrome. Zentralbl Allg Pathol 1981;125:364-8.

12. Barros MAE, Borges MF, Menezes FOM, Mantovani EA, Botelho RV, Adad SJ. Diabetes pancreático fibrocalculoso: uma forma rara de diabetes na América do sul. Relato de um caso. Arq Bras Endocrinol Metab 1993;37:34-6.

13. Polack FP, Transue DJ, Belknap WM. Transiet elevation of sweat chloride concentration in a malnourished girl with the Mauriac's syndrome. J Pediatr 1995; 126:261-3.

14. Harber EP, Curi R, Carvalho CRO, Carpinelli AR. Secreção da insulina: Efeito autócrino da insulina e modulação por ácidos graxos. Arq Bras Endocrinol Metab 2001;45:219-27.

15. Pashkova VS, Filippova LA, Bobrovskikh MP. Secondary glycogenosis (Mauriac's syndrome) in a young man with diabetes mellitus. Arkh Patol 1994;56:79-80.

16. Calil GF, Macdevit HO. Insulin-dependent diabetes mellitus: The initial lesion. N Engl J Med 1981;304:1454-65.

17. Mauras N, Merimee T, Rogol AD. Function of growth hormone-insulin-like growth factor I axis in the profoundly growth-retarded diabetic child: evidence for defective target organ responsiveness in the Mauriac's syndrome. Metabolism 1991;40:1106-11.

18. Huge-Jones P. Diabetes in Jamaica. Lancet 1955;29:891-7.

19. Mohan V, Nagalatimith SJ, Yajinik CS. Fibrocalculous pancreatic diabetes. Diabetes Metab Rev 1998; 14:153-70. 
20. Nwokolo C, Oli J. Pathogenesis of juvenile tropical pacreatitis syndrome. Lancet 1980;1:456-8.

21. Traisman S, Traisman ES. Mauriac's syndrome revisited. Eur J Pediatr 1984; 142:296-7.

22. Diabetes control and complications trial research group. The effect of intensive treatment of diabetes on the development and progression of long-term complications in insulin-diabetes mellitus. N Engl J Med 1993; 329:977-86.

23. Daneman D, Drash AL, Lobes LA. Progressive retinopathy with improved control in diabetic dwarfism (Mauriac's sydrome). Diabetes Care 1981:4:360-5.

24. Boland EA, Grey M, Oesterle L. Continuous subcutaneous insulin infusion: a new way to lower risk of severe hypoglicemia, improve metabolic control and enhance coping in adolescents with type 1 diabetes. Diabetes Care 1999;22:1779-84.

\section{Endereço para correspondência:}

Frederico Fernandes Ribeiro Maia R. Nunes Vieira 299, ap.702

30.350-120 Belo Horizonte, MG e.mail: Fredfrm@hotmail.com 